\title{
Experimental Performance Characterization of a Novel Direct Current Cold Cathode Neutralizer for Electric Thruster Applications
}

\author{
A. Gurciullo* and A. Knoll ${ }^{\dagger}$ \\ Surrey Space Centre, Guildford, Surrey, GU2 7XH, United Kingdom \\ P. Bianco \\ Airbus Defence and Space, Portsmouth, Hampshire, PO3 5PU, United Kingdom
}

\begin{abstract}
The performance of a novel neutralizer for space applications based on a $\mathbf{E} \times \mathbf{B}$ discharge is presented. Preliminary tests were carried out with argon gas and flow rates in the range of 5-10 SCCM. Electrons were extracted through an orifice of diameter $1.8 \mathrm{~mm}$. The maximum extracted current versus input power reported was $2.4 \mathrm{~mA} / \mathrm{W}$. The total power input, given by the sum of discharge power plus the extraction power, was in the range of 40-90 W. During extraction tests, the discharge current was limited at 0.2 A due to limit in the cooling system. Future work will be focused on tests at various extraction orifice diameters and cathode materials. Ultimately, xenon and non-conventional gases would be tested as working gases.
\end{abstract}

\section{Nomenclature}

$\begin{array}{ll}\alpha & \text { Extraction efficiency } \\ \mathbf{B} & \text { Magnetic field density } \\ \mathbf{E} & \text { Electric field } \\ H & \text { Energy efficiency parameter, given by extracted current versus input power } \\ I_{\text {cathode }} & \text { Cathode current, or discharge current } \\ I_{\text {collector }} & \text { Collector current, or electron extraction current } \\ P_{\text {cathode }} & \text { Power supplied to the cathode, or discharge power } \\ P_{\text {collector }} & \text { Power supplied to the collector, or extraction power }\end{array}$

\section{Introduction}

Electric thrusters in use by the satellite industry are reliant on external cathodes to provide electrons to sustain the plasma discharge and to neutralize the outgoing ion beam. The conventional approach to neutralize the outgoing ion beam is through the use of Hollow Cathodes and Dispenser Cathodes, which provide electrons by thermionic emission from the cathode material surface. One of the limitations of this conventional approach is that the propellants must be high purity non-reactive gases, such as xenon, to avoid cathode poisoning. If impurities are introduced, in particular atmospheric contaminates, they react with the emissive material causing degradation of the thermionic emission properties over time. Therefore, non-conventional molecular propellants, such as water vapour and carbon dioxide, are precluded from electric propulsion applications due to interactions and degradation of the neutralizer material. Furthermore, even if the Hollow Cathodes can tolerate higher impurity levels than Dispenser Cathodes (still at $99.99 \%$ of purity), ${ }^{1}$ the operating temperature, hence the power required by the external cathode, has to be increased in order to

*Ph.D. Research Student, Surrey Space Centre, University of Surrey, Student Member AIAA.

${ }^{\dagger}$ Lecturer in Plasma Propulsion, Surrey Space Centre, University of Surrey, Member AIAA. 
sustain the thermionic emission and minimizing poisoning due to chemisorption. ${ }^{2}$ At the same time, if the satellite industry eventually wants to avoid the high production cost and cost fluctuations of xenon, ${ }^{3}$ there is a clear incentive to switch to alternative propellants. ${ }^{4}$ As a consequence, alternative neutralizers, which do not rely on hot emissive materials and thus are not affected by cathode poisoning, have been receiving much attention from the research community. ${ }^{5,6}$

The aim of this study is to develop a low-power neutralizer based on a discharge in crossed $\mathbf{E} \times \mathbf{B}$ fields, rather than on thermionic emissive materials. In addition, future plans include neutralizer optimization in order to enhance its operability with high impurity rates in the feed gas and, ultimately, with molecular, reactive propellants.

In this paper, the results of the first experiments with argon as the working gas are reported. In particular, the $I-V$ characteristic, the extraction efficiency and the energy efficiency graphs are described for flow rates of $5,7.5$, and 10 SCCM.

The neutralizer was designed and assembled within the Surrey Space Centre, UK, and the project is supported by Surrey Satellite Technology Ltd (SSTL).

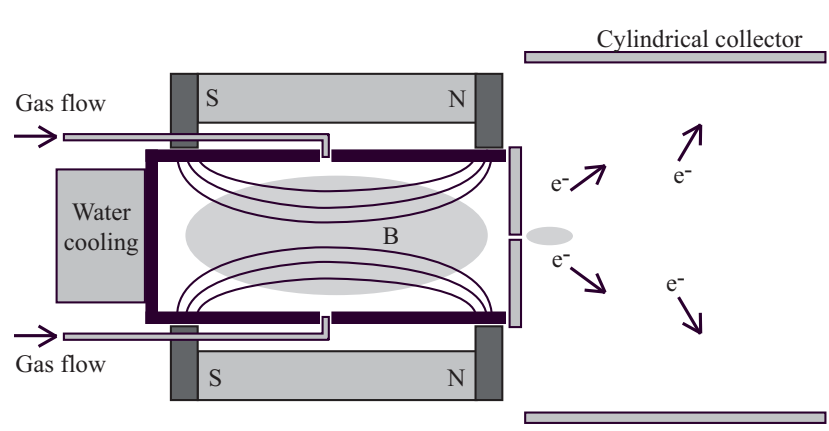

Figure 1: Schematic diagram of a conventional $\mathbf{E} \times \mathbf{B}$ electron source.

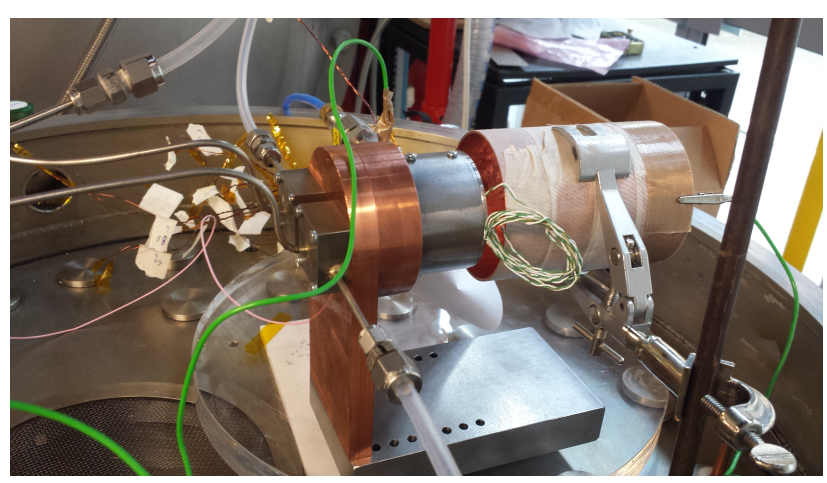

Figure 2: Experimental set-up.

A schematic diagram of a conventional $\mathbf{E} \times \mathbf{B}$ electron source and an external view of the neutralizer and the experimental set-up are showed in figures 1 and 2 . The design of the neutralizer is similar to the plasma electron source developed by Dostanko and Golosov. ${ }^{7}$ A stainless steel cathode encircles the volume in which the plasma discharge occurs, which is referred to as the discharge volume hereafter. The cathode area exposed to the plasma is about $18.74 \mathrm{~cm}^{2}$. An aluminium disk with a central orifice of diameter $1.8 \mathrm{~mm}$ is placed downstream of the discharge. A samarium-cobalt ring magnet creates the magnetic field which is directed inside the neutralizer by a highly permeable metal. The magnetic field is quasi-axial in the discharge volume with peak value of $700 \mathrm{G}$ and almost zero along the axis of axial-symmetry. To prevent overtemperature and failure of the magnet, a water cooling system is attached to the base of the neutralizer.

The cathode is connected to the negative potential output of a power supply. The disk is grounded and is electrically insulated from the cathode.

The working gas during the experiments reported in this paper was argon. The working was introduced inside the discharge volume through radial orifices made on the lateral wall of the cathode. The cathode potential was increased until the discharge initiated. Soon after the discharge is started, ions travel toward the cathode, while some electrons are trapped by the $\mathbf{E} \times \mathbf{B}$ fields. The trapped electrons collide and further ionize the working gas. The discharge is sustained either by collecting electrons at the grounded disk or by extracting electrons through the disk orifice.

The extraction of electrons from the discharge volume was accomplished using an external anode; a hollow cylindrical collector downstream of the extraction orifice. The collector inner diameter $(7.6 \mathrm{~cm})$ was larger than the neutralizer external diameter $(5.9 \mathrm{~cm})$, its axial length was $10 \mathrm{~cm}$, and it was positioned coaxially with the neutralizer. The hollow collector geometry prevented backflow of the working gas and the possible re-utilization of the neutral gas. The experiment set-up was installed inside a vacuum chamber which was de-pressurized by a diffusion pump. During the test campaign at different gas flow rates $(5,7.5$ and $10 \mathrm{SCCM}$ ) the background neutral pressure was within the range $9.7 \cdot 10^{-5}-1.5 \cdot 10^{-4}$ Torr.

The working gas flow rate was controlled by Bronkhorst EL-FLOW ${ }^{\circledR}$ Select model F-201CV mass flow 
controller. For each flow rate, measurements were recorded in order to study (i) the $I$ - $V$ characteristic of the neutralizer only, i.e. without electron extraction; (ii) the extraction efficiency,

$$
\alpha=I_{\text {collector }} / I_{\text {cathode }}
$$

where $I_{\text {collector }}$ is the electron current extracted and measured at the collector, and $I_{\text {cathode }}$ is the current supplied to the cathode; (iii) and, the energy efficiency, which is the ratio

$$
H=I_{\text {collector }} /\left(P_{\text {cathode }}+P_{\text {collector }}\right)
$$

where the denominator is the total power, given by the sum of the power needed to sustain the discharge, $P_{\text {cathode }}$, with the power needed to extract electron current, $P_{\text {collector }}$. It is important to note that the power supply of the cathode was controlled in current limited mode, which resulted in a much more stable discharge than in voltage limited mode.

\section{Results and Discussion}

The $I-V$ characteristic of the neutralizer only, i.e. without electron extraction, for various argon flow rates is showed in figure 3. During the experiment, it was noted that the minimum flow rate required for starting the discharge is 2 SCCM. As expected, at fixed discharge current, an increase of the flow rate causes a decrease of the discharge voltage. The cathode current was limited below $1 \mathrm{~A}$ (below $0.20 \mathrm{~A}$ for extraction tests) due to the risk of over-heating.

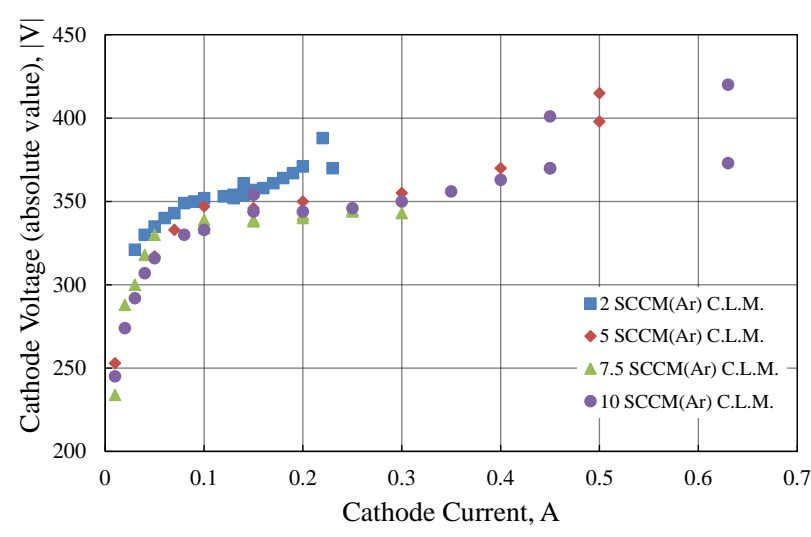

Figure 3: $I-V$ characteristic of the neutralizer only (no electron extraction) in current limited mode (C.L.M.) for various argon flow rates.

Three phases of the $I$ - $V$ characteristic can be noticed. At the lowest currents, a significant increase of the discharge voltage versus current is measured. This is followed by a region of slight rise of the voltage. At the highest currents, a second marked growth starts with an exponential profile. The $I$ $V$ characteristic at 2 SCCM reaches its upper limit at about $0.2 \mathrm{~A}$, after which the discharge becomes unstable and stops. This abrupt stop was not encountered at higher flow rates.

At 2 SCCM flow rate, electron current could not be collected from the external cylindrical collector: as soon as the collector extracted electrons from the neutralizer, the discharge perished. Therefore, the following data relative to the extraction performances are about flow rates of 5, 7.5, and 10 SCCM.

The plots of the extraction efficiency versus the collector voltage at various flow rates and cathode currents are shown in figures $4 \mathrm{a}-4 \mathrm{c}$. Figures $4 \mathrm{~d}-4 \mathrm{f}$ show the $I-V$ characteristics of the collector for various flow rates and cathode currents. Below $65 \mathrm{~V}$, electrons were not extracted. At voltage higher than $65 \mathrm{~V}$ the data appear following a linear trend or, at higher flow rates, a trend-line suggesting extraction saturation. Moreover, for a fixed cathode current, the slope of the plot increases at higher flow rates.In order to verify that the maximum collector current did not exceed the cathode current, i.e. the current of electrons created outside the neutralizer is not the main component of the collector current (which could lead to $\alpha>1$ ), the power supply of the collector was run in current limited mode. As soon as $I_{\text {collector }}$ approached $I_{\text {cathode }}$ trying to exceed it, the discharge inside the neutralizer extinguished. On the other hand, when the collector's power supply was run in voltage limited mode, above a threshold (about $100 \mathrm{~V}$, depending on the gas flow rate) the collector current matched the cathode current. In other words, the collector current is the extracted electron current.

The energy efficiency versus argon flow rate is shown in figure 5. It is important to note that, for each cathode current, data are for the highest extracted current recorded. For each flow rate in figure 5 , the three data points correspond to three highest data points showed in figures 4a (for 5 SCCM), 4c (for 7.5 SCCM), and 4c (for $10 \mathrm{SCCM}$ ). It can be noticed that the higher the extraction efficiency the higher the energy 


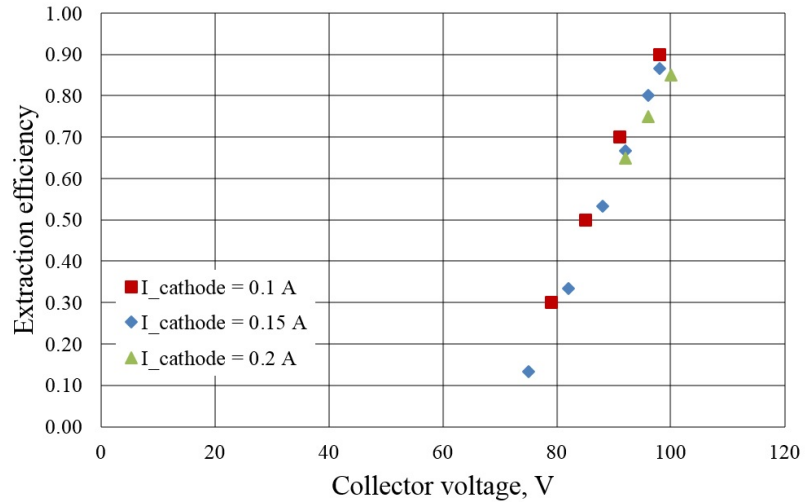

(a) $5 \mathrm{SCCM}$

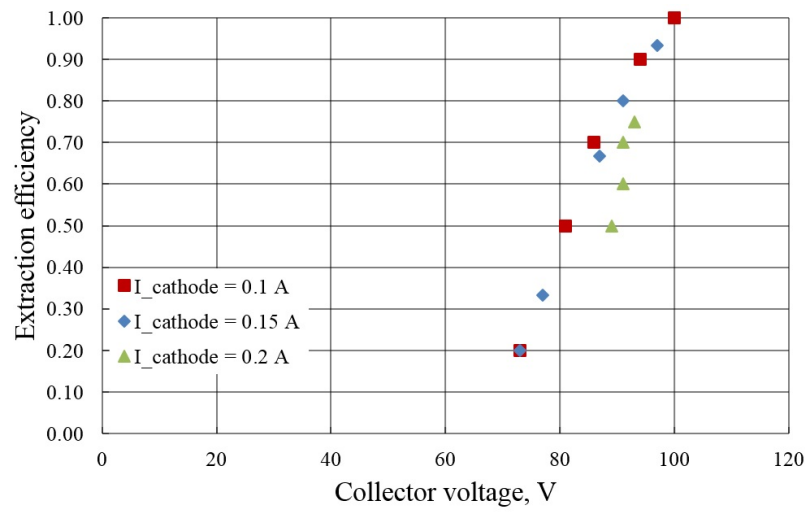

(b) $7.5 \mathrm{SCCM}$

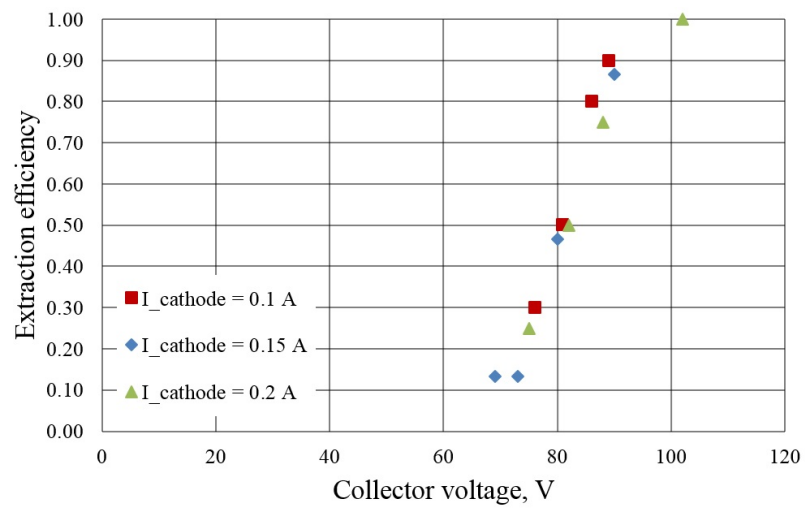

(c) $10 \mathrm{SCCM}$

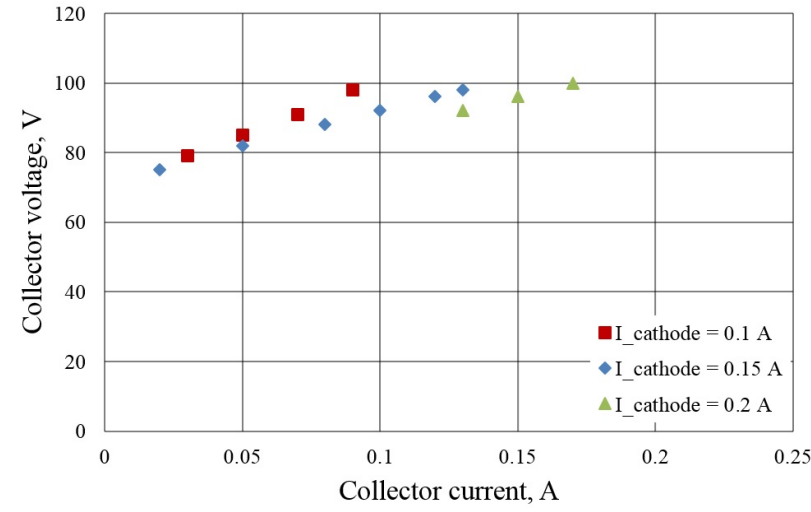

(d) $5 \mathrm{SCCM}$

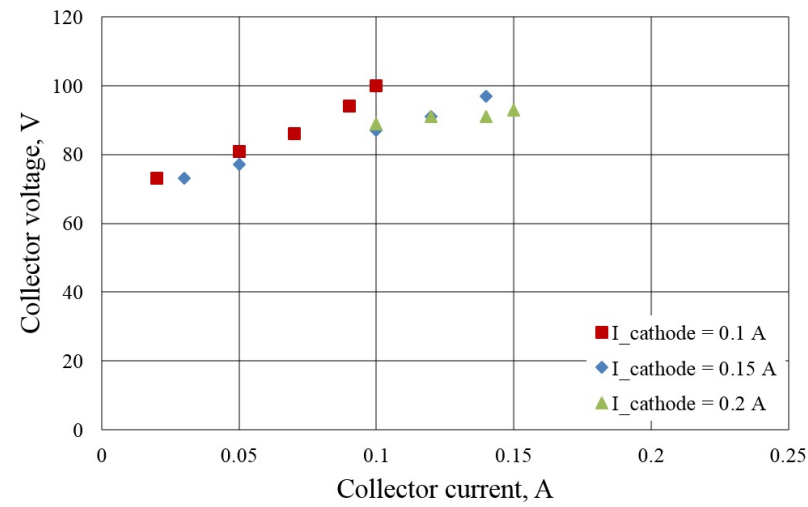

(e) $7.5 \mathrm{SCCM}$

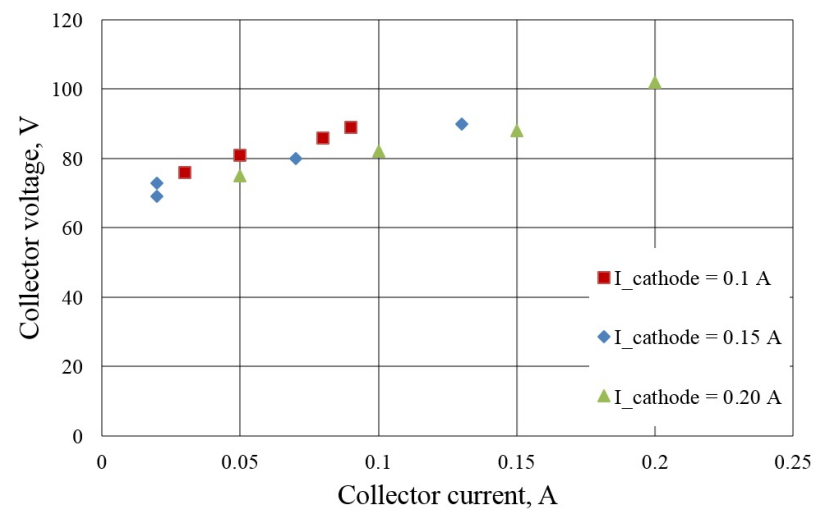

(f) $10 \mathrm{SCCM}$

Figure 4: Extraction efficiency vs collector voltage (figures 4a-4c) and $I-V$ characteristic of the collector (figures $4 \mathrm{~d}-4 \mathrm{f}$ ) for various cathode currents and argon flow rates.

efficiency. Interestingly, the upper limit of the energy efficiency tends toward $2.4 \mathrm{~mA} / \mathrm{W}$ for all three flow conditions, which is also the maximum value measured at full extraction at 10 SCCM. In figure 6 the trend of the maximum extracted current as a function of the total power for various argon flow rates is shown. Again, the plotted data points correspond to the data points at the highest extraction efficiency - for each cathode current - shown in figure 4a-4c. To note, the data are grouped in three clusters, depending on the cathode current from which electrons are extracted. It is expected that a well-studied choice of working gas-cathode material pair, together with an optimum extraction orifice diameter, would increase the energy efficiency of the neutralizer. ${ }^{7}$ 


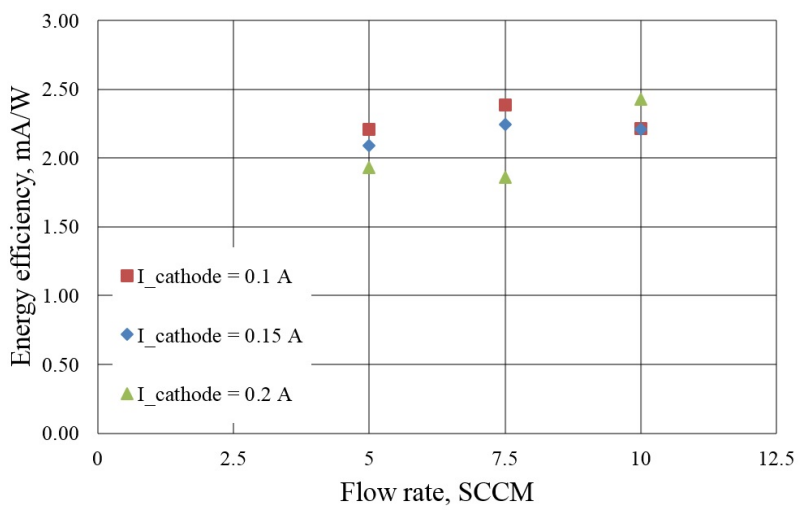

Figure 5: Energy efficiency versus argon flow rate for various cathode currents. Data are for the highest extracted current measured, which can be lower than the maximum extraction achievable.

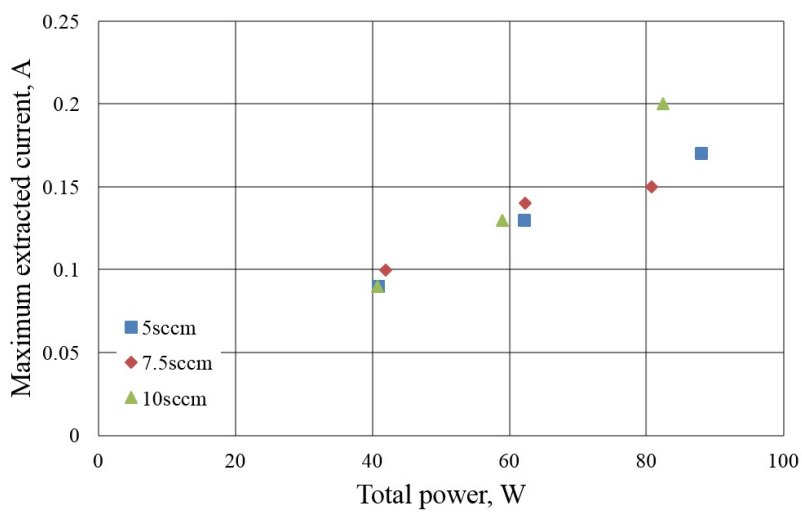

Figure 6: Maximum extracted current versus neutralizer power input for various flow rates. Data are for the highest extracted current measured, which can be lower than the maximum extraction achievable.

\section{Conclusion}

A novel neutralizer for low-power electric propulsion application has been fabricated and tested with argon as a working gas. The ultimate aim is to develop a neutralizer able to operate with high-impurity gases and molecular propellants. In this paper the results of the first tests were discussed. The discharge was sustained by crossed $\mathbf{E} \times \mathbf{B}$ fields, in which some of the electrons ionize the working gas while others are extracted through an orifice.

The tests have successfully demonstrated the extraction of electrons at relatively low input power. The neutralizer operated at total power input (discharge power plus extraction power) of 40-90 W for extracted current in the range of 0.1-0.2 A. The highest extracted current versus input power measured during the experiment campaign was $2.4 \mathrm{~mA} / \mathrm{W}$. Due to problem with the cooling system, the discharge current was held below $1 \mathrm{~A}$, and lower than $0.2 \mathrm{~A}$ during extraction experiments.

Future work is planned with other cathode materials and various extraction orifices. it is anticipated that an appropriate selection of cathode material together with the extraction diameter would optimize the energy efficiency for a specific working gas.

\section{References}

\footnotetext{
${ }^{1}$ Goebel D. M. and Katz I., Fundamentals of Electric Propulsion - Ion and Hall Thrusters, John Wiley \& Sons Inc, Hoboken, New Jersey, 2008, p. 304.

${ }^{2}$ Gallagher, H. E., "Poisoning of LaB6 Cathodes", Journal of Applied Physics, Vol. 40, No. 1, 1969, pp. 44-51. doi: $10.1063 / 1.1657092$

${ }^{3}$ Betzendahl R., "The 2014 Rare Gases Market Report," CryoGas International, July 2014, pp.28-30.

${ }^{4}$ Parissenti G., Koch N., Pavarin D., Ahedo E., Katsonis K., Scortecci F. and Pessana M., "Non Conventional Propellants for Electric Propulsion Applications", Space propulsion 2010 - 1841086, 2010.

${ }^{5}$ Weatherford B. R., Foster J. E. and Kamhawi H., "Visible Plume From a Low-Power ECR Waveguide", IEEE Transactions on Plasma Science, Vol. 39, No. 11, 2011, pp. 2942-2943 doi:10.1109/TPS.2011.2138722

${ }^{6}$ Jahanbakhsh S., Satir M. and Celik M., "Study of electron current extraction from a radio frequency plasma cathode designed as a neutralizer for ion source applications", Review of Scientific Instruments, Vol. 87, No. 2, 2016 doi:10.1063/1.4935015

${ }^{7}$ Dostanko, A. P. and Golosov, D. A., "Discharge and emission parameters of a plasma electron source based on a discharge in crossed $\mathrm{E} \times \mathrm{H}$ fields with various cathode materials", Technical Physics, Vol. 54, No. 10, 2009, pp.1454-1460. doi: 10.1134/S1063784209100089.

${ }^{8}$ Oks E., Plasma Cathode Electron Sources, WILEY-VCH Verlag GmbH \& Co. KGaA, Weinheim, Germany, 2006, Chap. 2 .
} 